\title{
Identification, release and olfactory detection of bile salts in the intestinal fluid of the Senegalese sole (Solea senegalensis)
}

\author{
Zélia Velez $\cdot$ Peter C. Hubbard $\cdot$ Kevin Welham • \\ Joerg D. Hardege $\cdot$ Eduardo N. Barata $\cdot$ \\ Adelino V. M. Canário
}

Received: 8 January 2009 / Revised: 16 April 2009 / Accepted: 17 April 2009 / Published online: 5 May 2009

(C) Springer-Verlag 2009

\begin{abstract}
Olfactory sensitivity to bile salts is wide-spread in teleosts; however, which bile salts are released in sufficient quantities to be detected is unclear. The current study identified bile salts in the intestinal and bile fluids of Solea senegalensis by mass spectrometry-liquid chromatography and assessed their olfactory potency by the electro-olfactogram. The main bile salts identified in the bile were taurocholic acid $(342 \mathrm{mM})$ and taurolithocholic acid $(271 \mathrm{mM})$ plus a third, unidentified, bile salt of 532.3 Da. These three were also present in the intestinal fluid (taurocholic acid, $4.13 \mathrm{mM}$; taurolithocholic acid, $0.4 \mathrm{mM}$ ). In sole-conditioned water, only taurocholic acid $(0.31 \mu \mathrm{M})$ was released in sufficient quantities to be measured (release rate: $24 \mathrm{nmol} \mathrm{kg}{ }^{-1} \mathrm{~min}^{-1}$ ). Sole had high olfactory sensitivity to taurocholic acid but not to taurolithocholic acid. Furthermore, olfactory sensitivity was higher in the upper (right) olfactory epithelium than the lower (left). These two bile acids contribute about $40 \%$ of the olfactory potency of intestinal fluid and account for the difference in potency at the two epithelia. Taurocholic acid (but not taurolithocholic
\end{abstract}

Z. Velez · P. C. Hubbard $(\varangle) \cdot$ E. N. Barata · A. V. M. Canário Centro de Ciências do Mar, Universidade do Algarve,

Campus de Gambelas, 8005-139 Faro, Portugal

e-mail: phubbard@ualg.pt

Z. Velez · E. N. Barata

Departamento de Biologia, Universidade de Évora,

Evora, Portugal

Z. Velez · J. D. Hardege

Department of Biological Sciences,

University of Hull, Hull, UK

K. Welham

Department of Chemistry, University of Hull, Hull, UK acid), and possibly other types of bile acid not tested, could be used as chemical signals and the upper olfactory epithelium is specialised for their detection.

KeyWords Olfaction · Asymmetry $\cdot$ Bile acid · Solea senegalensis $\cdot$ Flatfish
Abbreviations
EDTA Ethylene diamine tetra-acetic acid
EOG Electro-olfactogram
HPLC High-performance liquid chromatography
LC/MS Liquid chromatography/mass spectrometry
MS222 3-Aminobenzoic acid ethyl ester
$\beta$-NAD ${ }^{+} \quad \beta$-Nicotinamide adenine dinucleotide
SEM Standard error of the mean
TCH Taurocholic acid
TLC Taurolithocholic acid

\section{Introduction}

The main physiological function of bile acids and alcohols-steroidal compounds produced by the vertebrate biliary system-is to solubilise ingested lipids for easier digestion (e.g. Hofmann 1999). Fish, however, have also evolved a high olfactory sensitivity to this class of compound. This phenomenon is generally believed to be involved in chemical communication, conspecific recognition and/or identification of potential predators and prey (Døving et al. 1980; Hara 1994a, b; Sorensen and Caprio 1998; Zhang et al. 2001; Giaquinto and Hara 2008; Zhang and Hara 2009). Nevertheless, only in the sea lamprey Petromyzon marinus have clearly defined roles for bile acids as pheromones been established (Li et al. 2002; Sorensen et al. 2005). In teleosts, the most common bile salts are 
sulphated bile alcohols, mainly $5 \alpha$-cyprinol, 5 -chima-erol and $\mathrm{C}_{24}$ bile acids (cholic acid, chenodeoxycholic acid, deoxycholic acid and haemulcholic acid) and are conjugated mainly with taurine (Haslewood 1967). However, some glycine-amidated bile acids and, in some marine fish, cysteinolic bile acids have been identified (Une et al. 1991). In order to ultimately clarify the role of olfactory sensitivity to bile acids in fish, it is not only necessary to assess the sensitivity to different bile acids but also to identify those bile acids released in quantities sufficient to be detected by conspecifics and/or potential predators and prey (Zhang et al. 2001).

The Senegalese sole (Solea senegalensis), hereafter 'sole', is a benthic flatfish of the family Pleuronectidae. This fish undergoes a metamorphosis which involves a $90^{\circ}$ rotation of the body and the migration of the left eye to the right (upper) side. However, the left nostril, containing the olfactory epithelium and associated structures, does not migrate to the upper side but remains on the left (lower) side, facing the substratum (Rodríguez-Gómez et al. 2001). Thus, the two olfactory epithelia are exposed to different odour sources; this raises the possibility that the two olfactory epithelia have evolved differential sensitivity to odorants and there is functional asymmetry in the olfactory system. For example, the lower epithelium is more sensitive to aromatic amino acids, one of which, L-phenylalanine, is released by one of its prey species, the ragworm Hediste diversicolor (Velez et al. 2005, 2007b). This suggests that the lower epithelium is specialised for prey detection. It is possible that the upper epithelium, facing the water column, has been specialised mainly for intra-specific chemical communication and that bile acids are involved in this process; certainly, conspecific body-fluids are better detected by the upper epithelium than the by the lower (Velez et al. 2007a). Although the majority of bile acids released from the gall bladder is reabsorbed during intestinal transit, it has been shown that fish release some bile acids via the faeces (Zhang et al. 2001). The aim of the current study was, therefore, to identify the bile acids produced and released by the sole and assess their contribution to the olfactory potency of conspecific intestinal fluid and soleconditioned water on both upper and lower olfactory epithelia.

\section{Materials and methods}

Conspecific body-fluids and sole-conditioned water

Body-fluids (intestinal and bile fluid) were taken from adult sole (both sexes) that were being sampled as part of another study (Agulleiro et al. 2006). Bile fluid was taken directly from the gall bladder and intestinal fluid was extracted from the posterior $10 \mathrm{~cm}$ of intestine. Samples were pooled, diluted in distilled water (1:2), mixed thoroughly, centrifuged, aliquoted and frozen $\left(-20^{\circ} \mathrm{C}\right)$ until use. Sole-conditioned water (1 l) was taken from a 4001 tank in which six sole (average weight, $150 \mathrm{~g}$ ) were kept, unfed, in seawater (35\%) for 4 days. The water was filtered $(1.2 \mu \mathrm{m}$; Whatman GF/C filters) and all samples were then passed through reverse-phase C18 chromatography cartridges (IST-International Sorbent Technology, Hengoed, UK). Solid-phase extraction (SPE) was carried out according to the manufacturer's instructions. Briefly, cartridges were conditioned with methanol $(2 \mathrm{ml})$, washed with distilled water $(2 \mathrm{ml})$ and then the samples were applied. Finally, retained substances were eluted with $2 \mathrm{ml}$ methanol (eluate) and stored at $-20^{\circ} \mathrm{C}$ until use.

\section{High-performance liquid chromatography}

The intestinal fluid eluate was fractionated using a highperformance liquid chromatography (HPLC) system (Agilent 1100 series, Agilent Technologies, South Queensferry, West Lothian, UK) consisting of a quaternary pump, a degassing device, an auto-sampling injector, an automatic sample collector, a column oven with a cooling device necessary to keep the column temperature exactly at $28^{\circ} \mathrm{C}$ and a diode array detector scanning from 200 to $300 \mathrm{~nm}$. The column was an Ascentis C18 column $(25 \mathrm{~cm} \times 4.6 \mathrm{~mm}$, $5 \mu \mathrm{m})$. Samples were run with a linear gradient of water (pH 3.8) and acetonitrile (0-100\%) over $30 \mathrm{~min}$ and a flow rate of $0.7 \mathrm{ml} \mathrm{min}^{-1}$. Fractions were collected every $3 \mathrm{~min}$, evaporated under vacuum, dissolved in methanol, pooled and stored at $-20^{\circ} \mathrm{C}$ until use. Spectral data were collected and analysed with the software "Agilent ChemStation for LC and LC/MS system".

\section{Recording the electro-olfactogram}

The olfactory potency of bile salts, bile fluid, intestinal fluid and their respective HPLC fractions was assessed by the electro-olfactogram (EOG) as previously described (Velez et al. 2005). To reduce the electrical shunting effect of seawater, sole were adapted to dilute seawater $(12 \%$ ) over 4 days; this species naturally feeds and spawns in estuarine waters of reduced salinity (Cabral and Costa 1999; Cabral 2000; Anguis and Cañavate 2005). The fish were anaesthetised by immersion in water containing $100 \mathrm{mg}^{-1}$ MS222 (3-aminobenzoic acid ethyl ester, Sigma-Aldrich) followed by intra-peritoneal injection of $\operatorname{Saffan}^{\mathrm{TM}}$ $\left(300 \mu \mathrm{l} \times 100 \mathrm{~g}^{-1}\right.$ body-weight; Schering-Plough Animal Health, Welwyn Garden City, UK) and placed on a padded surface (with a slight backward tilt); aerated water (12\%o) was pumped over the gills (approximately $100 \mathrm{ml} \times 100 \mathrm{~g}$ body-weight ${ }^{-1} \mathrm{~min}^{-1}$ ) via a plastic tube inserted into the 
mouth. The body of the fish was covered by damp paper towel and the eyes covered with small pieces of black polythene. The upper olfactory rosette was exposed by cutting the overlying skin and musculature. The recording electrode was placed at a position that resulted in the largest response to the "standard" stimulus $\left(10^{-3} \mathrm{M}\right.$ L-cysteine $)$ and the reference electrode was placed lightly on the skin of the head nearby. The signal was digitised (DigiData 1322A, Molecular Devices Corporated, Sunny Vale, CA, USA) and stored on a computer running Axoscope ${ }^{\mathrm{TM}}$ software (version 9.2; Molecular Devices). All stimulants were dissolved directly in seawater of $12 \%$. At least $1 \mathrm{~min}$ was allowed between successive stimuli. The amplitude of the initial peak of the EOG was measured in millivolts, this was then blank-subtracted. Each amplitude was normalised to the amplitude of response to the "standard" stimulus $\left(10^{-3} \mathrm{M}\right.$ $\mathrm{L}$-cysteine). Although use of L-cysteine as a stimulus to find the best recording position may have biased the results, it is generally believed that there is little or no functional division in the olfactory epithelium of fishes (reviewed in Kasumyan 2004). Using the normalised EOG responses allows a direct comparison between the two olfactory epithelia, where absolute amplitudes are generally higher when recorded from the upper (Velez et al. 2005). Responses to the standard and blank were recorded at regular intervals throughout the session. The order in which odorants were presented was varied between fish, but individual odorants were presented in the order of increasing concentration. After all odorants had been tested on one olfactory epithelium (usually the lower) the fish was turned over and the same odorants were presented to the other epithelium.

Data treatment and statistical analysis

Responses to the standard and blank were recorded at regular intervals throughout the session. Responses of the two olfactory epithelia to each HPLC fraction were compared using Student's $t$-test for paired samples. Differences in responsiveness to an artificial mixture of bile acids based on the concentrations measures in bile and intestinal fluid between the upper and lower epithelia were then assessed by linear regression of log-transformed data (Hubbard et al. 2003) and comparing both the slopes and elevations of the regressions (Zar 1996). Unless otherwise stated, data are presented as mean \pm standard error of the mean (SEM). A $P$-value of $<0.05$ was taken to be statistically significant.

\section{Quantification of bile salts}

Bile salt concentration was measured using an enzymatic and fluorimetric approach described by Murphy et al. (1970). All chemicals (Tris-HCl buffer pH 7.2, EDTA, $\beta$ nicotinamide adenine dinucleotide $\left(\beta-\mathrm{NAD}^{+}\right), 3 \alpha$-hydroxy- steroid dehydrogenase from Pseudomonas testosteroni and hydrazine sulphate) were bought from Sigma-Aldrich (UK). All bile acids with a free $3 \alpha$-hydroxy group can be detected with the enzyme $3 \alpha$-hydroxysteroid dehydrogenase (Iwata and Yamasaki 1964); however, sulphated or glucuronidated bile acids require previous de-conjugation and solvolysis.

Enzymatic de-conjugation

The enzymatic de-conjugation was performed as described by Murphy et al. (1995). Briefly, the $\mathrm{pH}$ of each sample was adjusted to 5.6 (with $5 \mathrm{M} \mathrm{HCl}$ ), and placed in a water bath at $37^{\circ} \mathrm{C}$. After $10 \mathrm{~min}, 10 \mu \mathrm{l}$ choloylglycine hydrolase solution (100 units $\mathrm{mg}^{-1}$ protein; Sigma-Aldrich, UK) was added, and incubation continued overnight. Bile salts were then extracted by SPE as described above. The eluate was evaporated to dryness under nitrogen.

\section{Solvolysis}

After the enzymatic de-conjugation $1.8 \mathrm{ml}$ methanol, $1.4 \mathrm{ml}$ dimethoxypropane and $10 \mu \mathrm{l}$ concentrated $\mathrm{HCl}$ were added to each sample. After mixing, the liquid was left in the dark overnight. Samples were then evaporated to dryness, re-dissolved in water and bile salts were extracted by SPE (as above) and quantified by liquid chromatography/ mass spectrometry (see below).

\section{Liquid chromatography/mass spectrometry}

The HPLC conditions were identical to those previously described for sample fractionation. LC/MS analysis was carried out using a Thermo-Finnigan LCQ Classic ion trap mass spectrometer (Thermo-Finnigan, San Jose, CA, USA) and a binary liquid chromatography (LC) pump (Series 200, Perkin Elmer, UK). The mass spectrometer was operated in negative ion electrospray mode with a spray voltage of $4 \mathrm{kV}$. Nitrogen gas flows of 60 arbitrary units (sheath flow) and 20 arbitrary units (auxiliary flow) and a capillary temperature of $270^{\circ} \mathrm{C}$ were used to produce stable spray conditions. Data were collected in the full-scan mode, over the range $m / z, 150-1000$. 'Xcalibur' software (Thermo Scientific, UK) was used to process the mass spectral data and produce total ion chromatograms for the separation. Bile acids were identified by comparing the retention times and the mass spectra with standards, whenever possible. For those bile acids identified (taurocholic acid and taurolithocholic acid), standards at various concentrations $(5-500 \mu \mathrm{M})$ were subjected to the same procedure as the samples. Peak areas from the total ion chromatograms were plotted against the known concentrations from the standards to produce a calibration curve which was then used to estimate the concentrations in the samples. 


\section{Results}

Intestinal fluid

Out of the 10 HPLC fractions, the vast majority of olfactory activity was found only in the first 6 (Fig. 1a). At the upper olfactory epithelium, fractions 1 (0-3 min) and 4 (9$12 \mathrm{~min}$ ) were the most potent, each with about $40 \%$ of the total activity. At the lower epithelium, fraction 1 was the most potent. Both the unfractionated total and fraction 4 were more potent on the upper epithelium than the lower.

The largest amount of bile salts was detected in fraction 4 (Fig. 1b). Smaller amounts were detected in fractions 3 and 6. The LC-MS chromatogram of fraction 4 had several
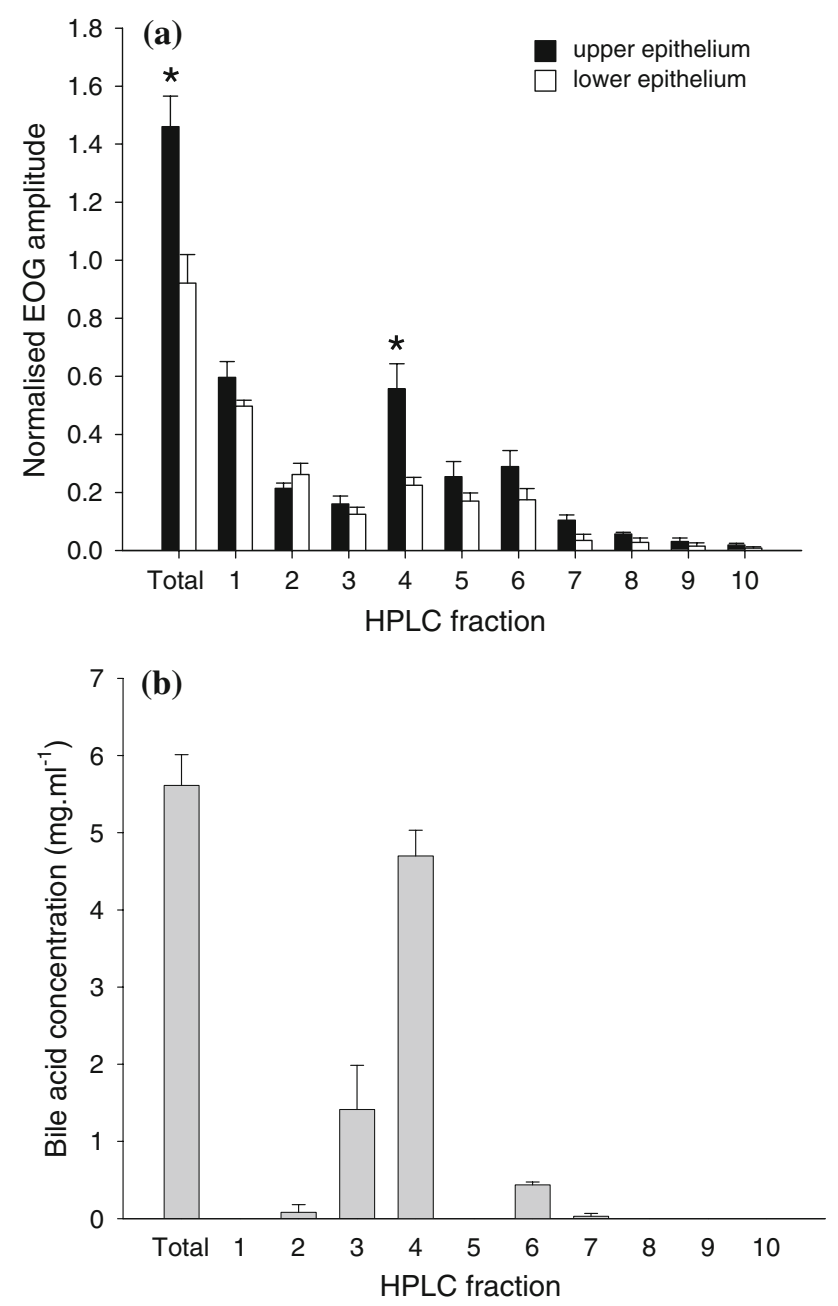

Fig. 1 Bile acids as odorants in the intestinal fluid of sole. a Olfactory responses of the upper (black bars) and lower (white bars) olfactory epithelia of sole to HPLC fractions of the C-18 eluate of conspecific intestinal fluid. Note that differences in olfactory potency between the two epithelia are found in the total and fraction 4 only. Data are shown as mean $\pm \operatorname{SEM}(n=5)$. $* P<0.05$. b Histogram showing the total bile salt concentration measured in each HPLC fraction (mean + SEM of triplicate measures). Note that the majority of bile salts are found in fraction 4 peaks. The first peak, with a retention time of $9.38 \mathrm{~min}$ and an apparent molecular peak at $\mathrm{m} / \mathrm{z} 515.3$ (Fig. 2a), was identified as taurocholic acid $(4.1 \mathrm{mM})$ by comparison with an authentic standard. The second peak, with a retention time of $9.97 \mathrm{~min}$ and an apparent molecular peak at $\mathrm{m} / \mathrm{z}$ 499.3 (Fig. 2b), was identified as taurolithocholic acid $(0.4 \mathrm{mM})$. The last well-defined peak, with a retention time of $10.56 \mathrm{~min}$ and an apparent molecular peak at $\mathrm{m} / \mathrm{z} 532.4$ (Fig. 2c), was not identified. Given its presence in fraction 4 , its molecular weight and mass spectrum, this compound is possibly a bile salt. The unfractionated eluate of the intestinal fluid had a mass spectrum similar to that of fraction 4, except for one extra peak (retention time $5.15 \mathrm{~min}$ ). The mass spectrum corresponding to this peak does not show the same peak profile as bile acids (Fig. 2d); for example, the peaks of double molecular mass present in Fig. 2a, b and c are absent in d. Also, a range of commercially available bile acids gave similar spectra to Figs. $2 \mathrm{a}-\mathrm{c}$ (data not shown). Thus, this unknown compound is unlikely to be a bile acid.

Olfactory sensitivity to taurocholic acid and taurolithocholic acid

The upper olfactory epithelium of sole had appreciable sensitivity to taurocholic acid, giving measurable responses down to $10^{-9}-10^{-8} \mathrm{M}$ (Fig. 3a). Due to the electrical shunting effect of the dilute seawater used, we suspect that this may be an underestimation of the true olfactory sensitivity, so thresholds of detection were not calculated. Nevertheless, the lower epithelium gave significantly smaller EOG responses, suggesting that this epithelium is less sensitive to taurocholic acid than the upper. However, neither epithelium showed any appreciable sensitivity to taurolithocholic acid (Fig. 3b). The olfactory activity of fraction 4 of the intestinal fluid eluate and the artificial mixture of bile acids at the same concentration as measured in this fraction are statistically equal (Fig. 3c); bile acids are likely responsible for the olfactory potency of fraction 4 .

Bile fluid

In the bile fluid, two main peaks were detected (Fig. 4a). The first had a retention time of $9.58 \mathrm{~min}$ and an apparent molecular peak at $\mathrm{m} / \mathrm{z}, 515.3$ and was identified as taurocholic acid, present at a concentration of $342 \mathrm{mM}$. The second peak had a retention time of $10.30 \mathrm{~min}$ and the mass spectrum showed the presence of taurolithocholic acid at $271 \mathrm{mM}$ and another, unidentified, bile acid with an apparent molecular peak at $m / z 532.3$ (Fig. 4b). This peak may correspond to the same unidentified peak detected in intestinal fluid. Another minor peak was present with a retention time of about 12.42 min with an apparent molecular peak at 
Fig. 2 Identification of bile acids in the intestinal fluid of sole. a LC-MS mass spectrum of the first peak detected in HPLC fraction 4 of the C- 18 eluate of intestinal fluid. This peak was identified as taurocholic acid. b LC-MS mass spectrum of the second peak detected in HPLC fraction 4. This peak was identified as taurolithocholic acid. c LC-MS mass spectrum of the minor peak detected in HPLC fraction 4 of intestinal fluid. This peak had a retention time of $10.6 \mathrm{~min}$ and an apparent molecular peak of $(\mathrm{m} / \mathrm{z}) 532.4$. The chemical identity is unknown. d Mass spectrum obtained by LC-MS of the first peak of the eluate of intestinal fluid sample. This peak had a retention time of $5.15 \mathrm{~min}$ and an apparent molecular peak of $(\mathrm{m} / \mathrm{z})$ 544.1. The chemical identity of this peak is unknown

Fig. 3 Olfactory sensitivity of sole to taurocholic acid (a) and taurolithocholic acid (b). Note that the upper epithelium gives significantly larger EOG responses to taurocholic acid (TCH) than the lower acid but neither of the epithelium shows appreciable sensitivity to taurolithocholic acid (TLC). Olfactory responses of the upper olfactory epithelium of sole to an artificial mixture of bile acids based on those identified in fraction 4 of the C-18 eluate of intestinal fluid and to fraction 4 itself (c). Data are shown as mean $\pm \operatorname{SEM}(n=5)$, ** $P<0.01$
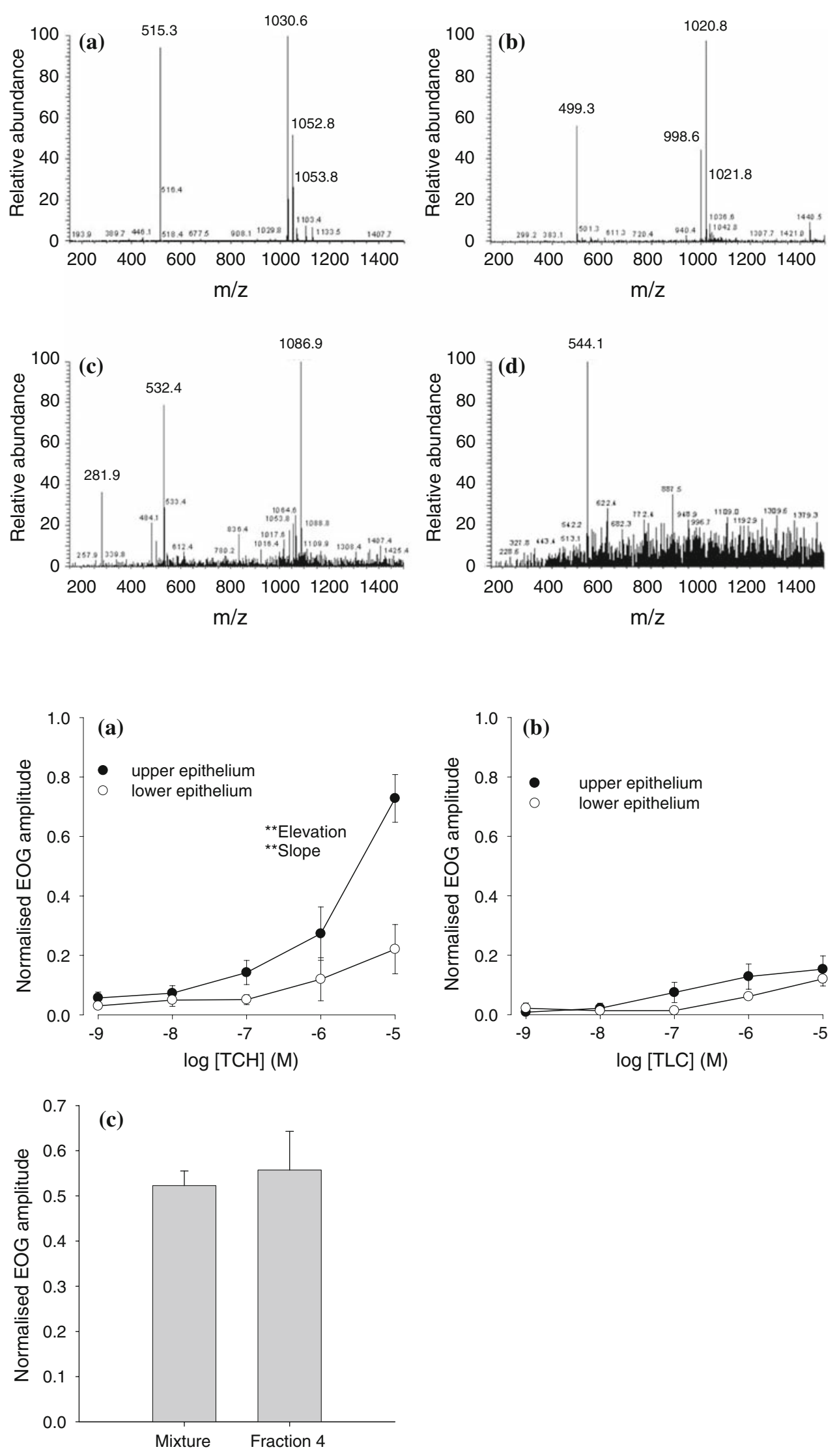
Fig. 4 LC-MS chromatograms of bile fluid. a Two main peaks detected (R.T 9.58 and 10.3) and a minor thirst peak with a R.T. of $12.42 \mathrm{~min}$. b LC-MS mass spectrum of the second peak (R.T. $10.3 \mathrm{~min}$ ) detected on the bile fluid sample. On this peak there are at least two different bile acids, the larger was identified as taurolithocholic acid and the smaller, unidentified, had an apparent molecular peak of $(\mathrm{m} / \mathrm{z})$ 532.4. c LC-MS mass spectrum of a peak with R.T. $12.4 \mathrm{~min}$ detected on the bile fluid sample. This peak had an apparent molecular peak of $(\mathrm{m} / \mathrm{z}) 516.3$. The chemical identity of this peak is unknown
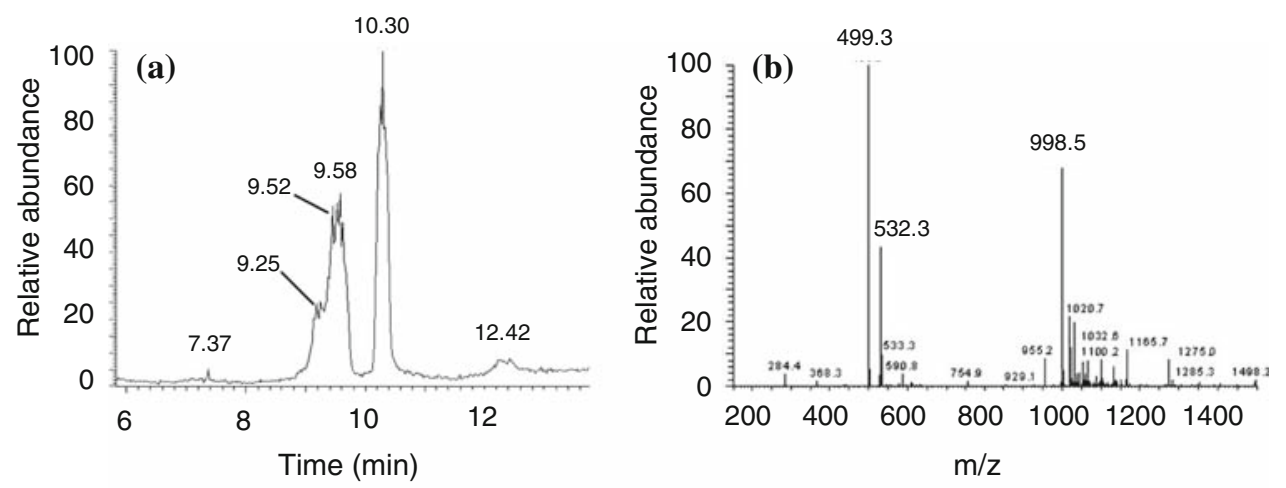

$\mathrm{m} / \mathrm{z} 516.3$ and a mass spectrum that likely corresponds to a bile acid (Fig. 4c).

\section{Sole-conditioned water}

The mass spectrum of sole-conditioned water showed two main peaks (Fig. 5a). The first had a retention time of $9.81 \mathrm{~min}$ and was identified as taurocholic acid $(0.31 \mu \mathrm{M})$. This gives an apparent release rate of $24 \mathrm{nmol} \mathrm{kg}{ }^{-1} \mathrm{~min}^{-1}$. The second peak had a retention time of $10.95 \mathrm{~min}$ and a mass spectrum that suggests the existence of at least three different bile acids, one of which was identified as taurolithocholic acid. Although detectable, the amount of taurolithocholic acid was insufficient to quantify. The other two

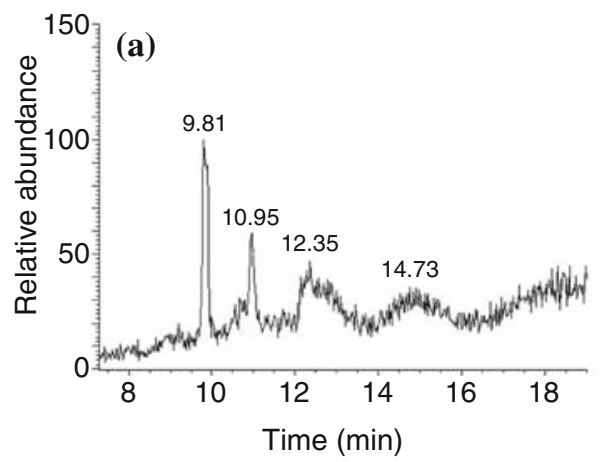

Fig. 5 LC-MS chromatograms of sole-conditioned water. a Two main peaks detected at retention times of 9.81 and $10.95 \mathrm{~min}$. b LCMS mass spectrum of the second peak detected in sole-conditioned water. This peak had a retention time of $10.95 \mathrm{~min}$ and corresponded putative bile acids show molecular peaks at $\mathrm{m} / \mathrm{z}, 557.1$ and 584.2 (Fig. 5b).

\section{Discussion}

Identification of bile acids

The current study shows that the main bile acids produced by the sole are taurocholic acid and taurolithocholic acid plus a minor third, unidentified, bile acid of $544.1 \mathrm{Da}$ molecular mass. Although the concentrations of taurocholic and taurolithocholic acids are comparable in the bile fluid, their concentrations in the intestinal fluid are markedly

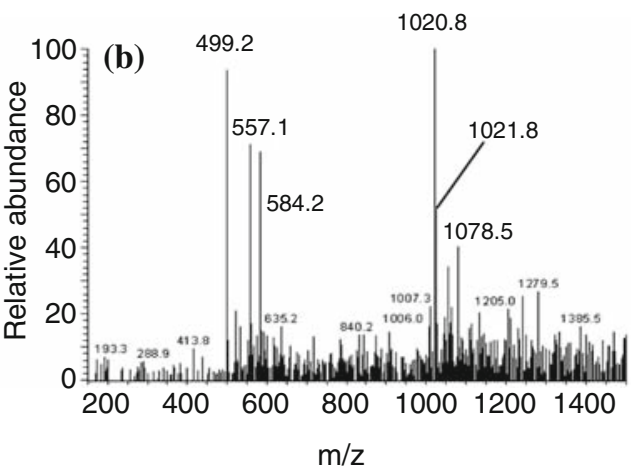

to three different bile acids, one of which was identified as taurolithocholic acid $(\mathrm{m} / \mathrm{z}$ 499.2). The other two unidentified peaks had apparent molecular peaks at $\mathrm{m} / \mathrm{z}, 557.1$ and $\mathrm{m} / \mathrm{z} 584.2$ 
different (10:1, taurocholic acid:taurolithocholic acid). This may reflect a higher re-absorption rate of taurolithocholic acid and/or transformation during intestinal transit; Zhang et al. (2001) noted a similar change in ratio of bile acids between bile and faeces in the lake char. Furthermore, taurocholic acid was the only bile acid released into the water in sufficient quantities to be measured; $24 \mathrm{nmol}$ $\mathrm{kg}^{-1} \mathrm{~h}^{-1}$ (cf. $4 \mathrm{nmol} \mathrm{kg}{ }^{-1} \mathrm{~h}^{-1}$ in the lake char; Zhang et al. 2001). This is matched by the olfactory sensitivity of sole to these bile acids; taurocholic acid is a significantly more potent odorant than taurolithocholic acid. The fraction containing most bile acids was also the fraction that evoked larger EOG responses from the upper olfactory epithelium than the lower and the artificial mixture of bile acids evoked similar amplitude EOGs to this fraction. Thus, it is likely that bile salts, taurocholic acid in particular, are responsible for the differential potency of intestinal fluid on the two epithelia. This is consistent with our hypothesis that the upper olfactory epithelium is specialised for the detection of conspecific-derived odorants. The third, unidentified, bile acid would not seem to make a significant contribution to the olfactory potency of intestinal or bile fluid. Nevertheless, it may still confer a particular quality to the overall odour released by the sole; this will only be clarified when it is identified and its olfactory potency assessed. Furthermore, other unidentified odorants in the intestinal fluid may be important; bile acids constitute only about $43 \%$ of the total olfactory activity of the intestinal fluid at the upper epithelium.

\section{Olfactory sensitivity to bile acids}

The role of olfactory sensitivity to bile acids in teleosts is not well understood. Bile acids are only produced by vertebrates (Haslewood 1967) so the majority of bile acids in the marine environment are likely to come from fish with a minor, and variable, fraction coming from marine mammals, reptiles and birds. The fact that sole produce, release and smell taurocholic acid is consistent with a role for this bile acid in chemical communication; the measured concentration of taurocholic acid in the sole-conditioned water is well within the detectable concentration range. However, other flatfish, such as the Japanese flounder (Paralichthys olivaceus) also produce taurocholic acid (Kim et al. 2007) and taurocholic acid was the main bile salt released by lake char (Zhang et al. 2001). Presumably, other componentswhether derived from the faeces or other sources (such as the mucus; Huertas et al. 2007; Velez et al. 2007a)—would be required to confer species-specificity to the odour. Alternatively, the meaning of the chemical message may depend on context or ratio or timing. Nevertheless, taurocholic acid is not the only bile acid that sole can smell; they also have sensitivity to sulphated $\mathrm{C}_{27}$ bile salts $5 \beta$-scymnol sulphate and $5 \alpha$-cyprinol sulphate (Velez et al. 2007a) produced by other fish species (Haslewood 1967; Goto et al. 2003). Thus, olfactory sensitivity to bile acids is not confined to those produced by conspecifics. Haslewood (1967) proposed an evolution from $\mathrm{C}_{27} 5 \alpha$-alcohol sulphates to $\mathrm{C}_{24} 5 \beta$ acids throughout the vertebrate lineage. Given that the role of bile salts as chemical signals occurred soon after, or even concurrently with, their role in lipid digestion (as seems likely given the use of bile salts as pheromones by the sea lamprey; Li et al. 2002; Sorensen et al. 2005), then it is possible that teleosts retained the olfactory receptor mechanisms for bile salts that they themselves no longer produced in order to recognise other species-potential predators, prey or competitors. However, as the current study makes clear, in investigating this type of question it is important to verify which bile salts are actually released to the water (and are therefore available for olfactory detection) rather than simply which bile salts are present in the bile fluid (Zhang et al. 2001). Furthermore, the function of olfactory sensitivity to hetero-specific derived bile salts needs clarification.

\section{Summary}

In summary, the main bile salts produced by the sole are taurocholic acid and taurolithocholic acid plus a minor unidentified bile salt (532.3 Da). Taurocholic acid is released via the faeces, where it constitutes about $40 \%$ of the olfactory activity, to the water in sufficient quantities to be detected by the olfactory system of conspecifics, especially by the upper (right) olfactory epithelium. These results suggest that taurocholic acid may be used as a chemical signal by the sole and that the upper epithelium is specialised for this function.

Acknowledgments This study was financed by grants SFRH/BD/ $16242 / 2004$ and POCTI/CVT/38831/2001 from the Portuguese National Science Foundation and the European Union Social Funds for Regional Development. The authors wish to thank Drs. Neil Duncan, Joan Cerdà and Mar Huertas (Center of Aquaculture-IRTA, Tarragona, Spain) for collecting bile and intestinal fluids from sole. The experiments described herein comply with the "Principles of animal care" (No. 86-23, revised 1985) of the National Institute of Health (USA) and Portuguese national laws.

\section{References}

Agulleiro MJ, Anguis V, Cañavate JP, Martínez-Rodríguez G, Mylonas CC, Cerdà J (2006) Induction of spawning of captivereared Senegal sole (Solea senegalensis) using different administration methods for gonadotropin-releasing hormone agonist. Aquaculture 257:511-524. doi:10.1016/j.aquaculture.2006.02. 001

Anguis V, Cañavate JP (2005) Spawning of captive Senegal sole (Solea senegalensis) under a naturally fluctuating temperature regime. Aquaculture 243:133-145 
Cabral HN (2000) Comparative feeding ecology of sympatric Solea solea and $S$. senegalensis, within nursery areas of the Tagus estuary, Portugal. J Fish Biol 57:1550-1562

Cabral H, Costa MJ (1999) Differential use of nursery areas within the Tagus estuary by sympatric soles, Solea solea and Solea senegalensis. Environ Biol Fishes 56:389-397

Døving KB, Selset R, Thommesen G (1980) Olfactory sensitivity to bile acids in salmonid fishes. Acta Physiol Scand 108:123-131

Giaquinto PC, Hara TJ (2008) Discrimination of bile acids by the rainbow trout olfactory system: evidence as potential pheromones. Biol Res 41:33-42

Goto T, Holzinger F, Hagey LR, Cerrè C, Ton-Nu H-T, Schteingart CD, Steinbach JH, Shneider BL, Hofmann AF (2003) Physicochemical and physiological properties of $5 \alpha$-cyprinol sulfate, the toxic bile salt of cyprinid fish. J Lipid Res 44:1643-1651

Hara TJ (1994a) The diversity of chemical stimulation in fish olfaction and gustation. Rev Fish Biol Fish 4:1-35

Hara TJ (1994b) Olfaction and gustation in fish: an overview. Acta Physiol Scand 152:207-217

Haslewood GAD (1967) Bile salt evolution. J Lipid Res 8:535-550

Hofmann AF (1999) Bile acids: the good, the bad, and the ugly. News Physiol Sci 14:24-29

Hubbard PC, Barata EN, Canário AVM (2003) Olfactory sensitivity to catecholamines and their metabolites in the goldfish. Chem Senses 28:207-218

Huertas M, Hubbard PC, Canário AM, Cerdà J (2007) Olfactory sensitivity to conspecific bile fluid and skin mucus in the European eel Anguilla anguilla (L). J Fish Biol 70:1907-1920

Iwata T, Yamasaki K (1964) Enzymatic determination and thin-layer chromatography of bile acids in blood. J Biochem 56:394-424

Kasumyan AO (2004) The olfactory system in fish: structure, function, and role in behaviour. J Ichthyol 44(Suppl 2):S180-S223

Kim S-K, Matsunari H, Takeuschi T, Yokoyama M, Murata Y, Ishihara K (2007) Effect of different dietary taurine levels on the conjugated bile acid composition and growth performance of juvenile and fingerling Japanese flounder Paralichthys olivaceus. Aquaculture 273:595-601. doi:10.1016/j.aquaculture.2007.10.031

Li W, Scott AP, Siefkes MJ, Yan H, Liu Q, Yun S-S, Cage DA (2002) Bile acid secreted by male sea lamprey that acts as a sex pheromone. Science 296:139-141

Murphy GM, Biling BH, Baron DN (1970) A fluorimetric and enzymatic method for the estimation of serum total bile acids. J Clin Pathol 23:594-598
Murphy GM, Maghsoudloo M, Qureshi MY (1995) Assay of bile acids in biological samples. In: Makin HLJ, Gower DB, Kirk DN (eds) Steroid analysis. Blackie Academic and Professional, Glasgow, pp 527-560

Rodríguez-Gómez FJ, Rendón-Unceta C, Sarasquete C, Muñoz-Cueto JA (2001) Distribution of neuropeptide Y-like immunoreactivity in the brain of the Senegalese sole (Solea senegalensis). Anat Rec 262:227-237

Sorensen PW, Caprio J (1998) Chemoreception. In: Evans DH (ed) The physiology of fishes, 2nd edn. CRC Press, Boca Raton, pp 375-405

Sorensen PW, Fine JM, Dvornikovs V, Jeffrey CS, Shao F, Wang J, Vrieze LA, Anderson KR, Hoye TR (2005) Mixture of new sulfated steroids functions as a migratory pheromone in the sea lamprey. Nat Chem Biol 1:324-328

Une M, Goto T, Kihira K, Kuramoto T, Ki Hagiwara, Nakajima T, Hoshita $\mathrm{T}$ (1991) Isolation and identification of bile salts conjugated with cysteinolic acid from bile of the red seabream, Pagrosomus major. J Lipid Res 32:1619-1623

Velez Z, Hubbard PC, Barata EN, Canário AVM (2005) Evidence for functional asymmetry in the olfactory system of the Senegalese sole (Solea sensgalensis). Physiol Biochem Zool 78:756-765

Velez Z, Hubbard PC, Barata EN, Canário AVM (2007a) Differential detection of conspecific-derived odorants by the two olfactory epithelia of the Senegalese sole (Solea senegalensis). Gen Comp Endocrinol 153:418-425. doi:10.1016/j.ygen.2007.02.016

Velez Z, Hubbard PC, Hardege JD, Barata EN, Canário AVM (2007b) The contribution of amino acids to the odour of a prey species in the Senegalese sole (Solea senegalensis). Aquaculture 265:336342. doi:10.1016/j.aquaculture.2007.02.029

Zar JH (1996) Biostatistical analysis. Pearson Higher Education, New Jersey

Zhang C, Hara TJ (2009) Lake char (Salvelinus namaycush) olfactory neurons are highly sensitive and specific to bile acids. J Comp Physiol A 195:203-215. doi:10.1007/s00359-008-0399-y

Zhang C, Brown SB, Hara TJ (2001) Biochemical and physiological evidence that bile acids produced and released by lake char (Salvelinus namaycush) function as chemical signals. J Comp Physiol B 171:161-171. doi:10.1007/s003600000170 